\title{
Using environmental affordances to direct people natural movement indoors
}

\author{
Elisângela Vilar ${ }^{1 *}$, Luís Teixeira ${ }^{1}$, Francisco Rebelo ${ }^{1}$, Paulo Noriega $^{1}$ and Júlia Teles ${ }^{2}$ \\ ${ }^{1}$ CIPER / Ergonomics Laboratory, FMH-Technical University of Lisbon, Estrada da Costa 1499-002, Cruz \\ Quebrada - Dafundo, Portugal \\ ${ }^{2}$ CIPER / Mathematics Unit, FMH-Technical University of Lisbon, Estrada da Costa 1499-002, Cruz Quebrada- \\ Dafundo, Portugal
}

\begin{abstract}
Understanding and predicting people's displacement movement is particularly important for professionals involved in planning complex buildings (e.g., hospitals, convention centers, subway stations and university campus). Some decisions taken by the visitors while choosing what route to follow can be influenced by some environmental cues which can act as a factor of attraction, influencing the wayfinding process. Therefore, the main objective of this study was to evaluate the hypotheses that, in the context of a simulated emergency egress, people prefer to move along either a wider and with more lighting corridor or to bear right. To collect the users' responses, a constant stimulus method was used, combined with a twoforced choices method, involving the projection of stereoscopic images in a wall-screen. Results suggest that, in a "T-type" intersection, users randomly chose which direction to follow. However, if there is an increment in the width of the side corridor, users tend to follow the wider corridor. When light is inserted, users also prefer to choose the corridors with more lighting. In situations where the variables corridor width and existence of lighting are concurrent, the corridors with light are the most chosen by the users.
\end{abstract}

Keywords: wayfinding, decision-taking, affordance, virtual reality

\section{Introduction}

When complex buildings (e.g., hospitals, convention centers and university campus) are the focus of intervention by professionals involved in planning these structures, is particularly important to predict people's movement within built environments. According to Conroy [1], these professionals are not able to know precisely how people displacement occurs in a built environment, neglecting this information and replacing it by intuition. This gap in the knowledge about people's behavior regarding to their navigation indoors may contribute to increase the wayfinding problems, with which the visitors are often confronted while interacting with complex buildings.

An approach to study the people's displacement indoors can be based on the affordances that the environment furnishes or affords the observer [2]. Some authors state that affordances are environmental properties that have some meaning to guide the observer's behavior [3,4]. The conscious use of this concept in architecture is based on the definition of a set of variables, which express the different priorities and capabilities of users under various conditions, creating congruence between what people realize they can do and the activities they can really perform. The formulation of this knowledge might be defined by studying people's interaction behavior with indoor environments, mainly with regard to the decisions taken under certain conditions (e.g., different corridors intersection's types, emergency $v s$. normal situation).

In this way, the main objective of this study is to investigate affordances of indoor corridors, namely the influence of architectural variables (i.e., corridor

*Corresponding author. E-mail: elipessoa@gmail.com 
width and lighting) and the right/left bias in people's decision-taking during the interaction with this environment in an emergency egress. It aims to assess if these variables can be considered a factor of attraction to increase the corridors' affordance, using a set of virtual indoor hallways. It is assumed that such factors of attraction, if they are proved as such, will significantly influence people's travel decisions during an emergency egress. The main hypotheses are that people prefer to follow: (1) the wider corridors; (2) the corridors with more lighting, and (3) considering a neutral situation and based in previous studies results [e.g., 5, 6] it is expected that, in a "T-type" intersection users tend to turn right.

\section{Methodology}

For this study, a set of static images of virtual indoor hallways was presented to participants using a stereoscopic projector and 3D shutter glasses. To collect the participants' responses, the images' sequence was presented using a constant stimulus method combined with a two-forced choice method.

The participants' circulation along simulated corridors (i.e., escape routes) was the study's main focus, with special attention to their decision-taking (i.e., path selection) at the corridors' intersection points. As such, these concerns have conditioned the architecture of the experimental virtual environment designed for this study.

\subsection{Design of the experiment}

In order to assess the influence of the independent variables - corridor width and lighting - on the path's selection by participants in a simulated emergency egress, three situations were considered: corridors width with same lighting, lighting in wider corridors and lighting in narrower corridors. Considering this, 27 experimental conditions representing indoor situations formed by two corridors linked by a "T-type" intersection were designed, providing two alternative arms or directional choices (Figure 1). Thus, the participants were assigned to a setting which was composed of a main corridor that ends in a perpendicular corridor with two side corridors (i.e., an alternative hallway to where the user could turn), offering two alternative paths (i.e., to turn left or to turn right).

The width of the side corridor was manipulated to test the influence of the corridor's width on the route decisions. Thus, the main corridor's width was fixed at $2 \mathrm{~m}$ wide, but the perpendicular alternative corridors (left and right) had their width increased in $0.5 \mathrm{~m}$, from $2 \mathrm{~m}$ until reaching $4 \mathrm{~m}$ wide each. As a result, nine stimuli were obtained, corresponding to the corridors $\mathrm{C} 1$ - $\mathrm{C} 9$ in Figure 1.

The existence of lighting was also manipulated to test the influence of this variable on participants' decision about the path to follow. Thus, lighting was added in the wider corridors and eight stimuli were obtained through this (i.e., corridors C11 - C18 in Figure 1). In order to verify which variable had more influence in participants' decision, eight stimuli were created with lighting in narrower corridors, designing a situation that confronts narrower and with more lighting $v s$. wider and darker corridors. These stimuli can be seen in Figure 1 as corridors C20 - C27. The influence of lighting regardless the corridor width was verified through two stimuli in which lighting was placed in the left and in the right corridor when both had the same width (i.e., $2 \mathrm{~m}$ wide). Those stimuli are represented in Figure 1 as corridors C10 and $\mathrm{C} 19$.

An example of the virtual environments developed from those conditions can be seen in Figure 2.

The stimuli were presented according to the method of constant stimuli, and a method of forced choice between two alternatives was used to collect answers. All the participants were exposed to 2 blocks of 112 trials, in a randomized sequence, in which all of the stimuli (except corridor C1) were repeated 8 times. The corridor $\mathrm{C} 1$ represents the neutral condition and was considered to verify the right/left bias in the corridor's choice. This corridor was repeated 16 times in order to collect more responses related to this issue. The second block had the trials organized in the inversed order used in the first block. To exclude an eventual sequence effect, each half of the sample was assigned, in first place, to one of the blocks.

The inter-stimulus interval varied from 800 to $1000 \mathrm{~ms}$ and the stimulus maximum duration was $1400 \mathrm{~ms}$, but it could be less because in the moment that the participant pushed the button to select an answer (i.e., a direction), the corridor's image disappeared and an inter-stimulus screen was presented. The inter-stimulus screen was a gray screen with the image of a black cube in the center.

Participants were unaware of the real objective of the experiment and were asked to act in a realistic/natural manner in order to evaluate a new system for virtual reality (VR) simulation. They were told that they should choose one of the available 
paths as fast as possible, since they were in an emergency situation.

\subsection{Virtual environment (VE)}

The VE used in this study, according to the experimental conditions previously defined, was generated based on the following requirements, which resulted from systematic meetings involving experts in Ergonomics, Architecture, Psychology, Design and Computer Engineering:

- Existence of a point of decision-taking related to which route to follow, with two alternative paths (left or right) - to use of a "T-type" intersection;

- Maintain the decision point - to use the same distance, from the starting point until the decision point for all stimuli;

- Constant width of the main corridor for all stimuli;

- Variable width in the alternative paths (left or right corridor adding $0.5 \mathrm{~m}$ as width increment);

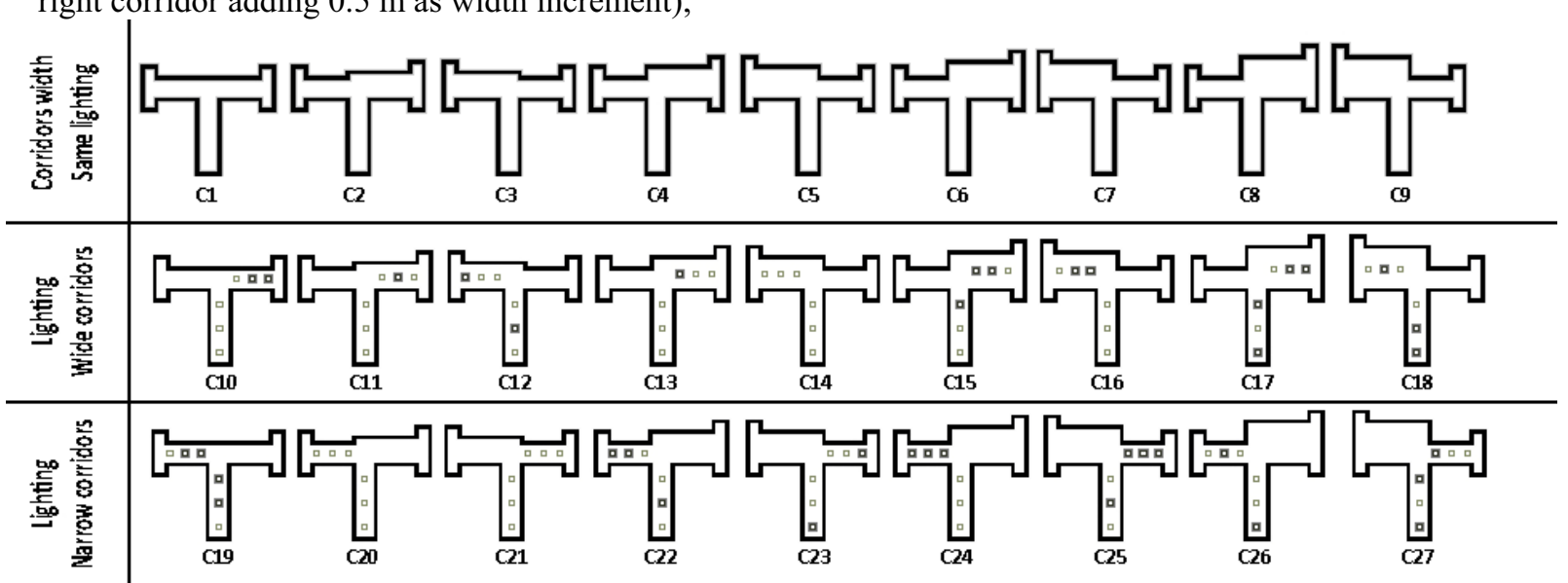

Figure 1

Twenty-seven different corridors according to the studies' independent variables

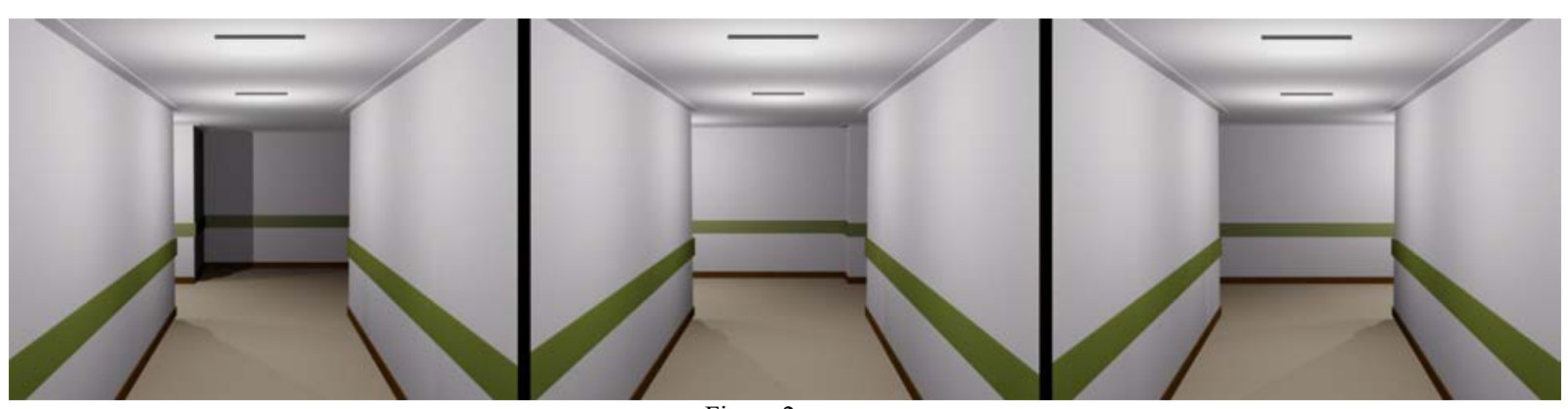

Figure 2

Examples of the images of corridors presented to the participants. In the left image there is an example of the narrower corridor with more lighting $v s$. the wider and darker corridor. The middle image has an example of a situation in which the lighting is in the wider corridor. The right image show the situation where left and right corridors have the same width but the right corridor has more lighting.
- Uniform light for width experimental condition - without light/shadows;

- Create light/shadow effects for experimental conditions which considered lighting;

- Avoid extra environmental cues - to use non identifiable/visible textures in the walls or floor, to use solid colors instead;

- Insert decorative elements to increase depth perspective - to include ceiling moldings, wainscoting and baseboards. 
The development of the VE was performed following various phases. First, 2D plans, which were the base structure of the VEs, were designed using the software Autodesk ${ }^{\circledR}$ AutoCAD 2009. The length $(7 \mathrm{~m})$ and the width $(2 \mathrm{~m})$ of the main corridor was maintained constant for all conditions, while the lateral corridor (left or right) had an increment of $0.5 \mathrm{~m}$ over the width. These $2 \mathrm{D}$ plans were exported to Autodesk ${ }^{\circledR}$ 3D Studio Max 2009 in order to model the $3 \mathrm{D}$ environments. In this phase, some elements such as colors of the walls and floor, ceiling moldings, wainscoting and baseboards - were inserted in the corridors to increase their realism level. Later, a free plugin (OgreMax v1.6.23) exported the environment which were presented by the ErgoVR system [7], developed by the Ergonomics Laboratory at $\mathrm{FMH}-\mathrm{Technical}$ University of Lisbon.

\subsection{Experimental settings}

A Lightspeed DepthQ 3D video projector and a MacNaughton Inc's APG6000 active glasses comprised the VR system used for the experimental tests. A Thrustmaster FireStorm Dual Analogue 3 Gamepad was used as an input device, in order to collect the participants' answers. Participants were asked to press the Gamepad's functional buttons on the right, according to the chosen direction (i.e., left and right).

The projected image size was $1.72 \mathrm{~m}$ (horizontal) by $0.95 \mathrm{~m}$ (vertical) with an aspect ratio of 16:9. The observation distance (i.e., the distance between the observers' eyes and the screen) was $1.50 \mathrm{~m}$, resulting in a $35.2^{\circ}$ of vertical field-of-view (FOV) and $59.7^{\circ}$ of horizontal FOV. All participants remained standing during the experimental session at the same location (marked on the floor) to ensure the same observation distance.

\subsection{Participants}

Eleven subjects, one male and ten females, aged between 20 and 68 years old (mean age $=36$ years; $\mathrm{SD}=14.59)$ participated as volunteers in this pilot study. Ten participants declared, through a questionnaire, to be right-handed and one declared to be left-handed. All participants had normal sight or had corrective lenses. They also reported no physical or mental conditions that would prevent them from participating in a VR simulation. Participants were alternatively assigned to see, firstly, the first sequence, followed by the second sequence, or the inverse order.

\subsection{Procedure}

The experimental session was comprised by a training stage and an experimental test.

Before starting, all participants were asked to sign a form of consent and advised they could stop the experimental session at any time they wanted. The average duration of each experimental session was 20 minutes.

The experimental session started with a training stage, in which some explanations about the experiment and the equipment involved were given to the participants. They also saw images of the intersection type and received instructions regarding to the task they were requested to fulfill. For the training stage participants were told that they should choose between two alternative paths, and that the selected path represents the one they would take if they were escaping from a building in an emergency egress. The training stage also comprised of two blocks using a sequence of images like those used in the experimental test. In the first block, participants were asked to point, with their hands, to each alternative corridor that they could see in the image. This procedure intended to ensure that the participants realized the alternative paths that they had in front of them. The second block intended to make participants familiar with: i) the command buttons in order to choose their direction and, ii) the time available for their answer.

The experimental test started after participants had given the required answers, in the time available, and had declared they felt confident and comfortable with the command buttons. For the experimental test, participants were assigned to the first sequence of 112 trials. When the first sequence was fulfilled, and after a five minute break, in the absence of simulator sickness symptoms, participants were assigned to the second sequence of 112 trials. At the end of the experimental test, a demographic questionnaire was applied to collect information such as age, gender, occupation and dominant hand. Participants were also asked to answer questions related to the experimental test. 


\section{Results and discussion}

The participants' choice in what concerns the direction, for each experimental condition, considering that they were escaping from a building in an emergency situation, is presented in Table 1. The results encompass the participants' directional choice, by experimental condition, for all trials, and for all participants (88 observations for each stimulus). All invalid answers were considered missing values.

All statistical analyses were performed with the software IBM SPSS v.19. The significance level considered was $5 \%$.

The main hypotheses of this study were that (1) people prefer to follow the wider corridors, (2) the corridors with more lighting, and (3) users tend to turn right.

Table 1

Global results for the participants' directional choices in the 27 stimuli for the three variables

\begin{tabular}{|c|c|c|c|c|c|c|c|}
\hline Variables & Corridor & Missing & Left & Right & $\begin{array}{l}\text { Total without } \\
\text { Missing }\end{array}$ & $\%$ Left & \% Right \\
\hline \multirow{9}{*}{$\begin{array}{l}\frac{5}{ \pm} \\
\frac{0}{3} \\
\vdots \\
\frac{0}{0} \\
\frac{0}{2}\end{array}$} & C1 & 6 & 61 & 109 & 170 & 35.9 & 64.1 \\
\hline & C2 & 1 & 11 & 76 & 87 & 12.6 & 87.4 \\
\hline & C3 & 1 & 60 & 27 & 87 & 67 & 31 \\
\hline & C4 & 0 & 10 & 78 & 88 & 11.2 & 88.6 \\
\hline & C5 & 0 & 65 & 23 & 88 & 74 & 26.1 \\
\hline & C6 & 0 & 11 & 77 & 88 & 12.5 & 87.5 \\
\hline & C7 & 1 & 79 & 8 & 87 & 90.8 & 9.2 \\
\hline & C8 & 1 & 14 & 73 & 87 & 16.1 & 83.9 \\
\hline & C9 & 0 & 69 & 19 & 88 & 78.4 & 21.6 \\
\hline \multirow{9}{*}{ 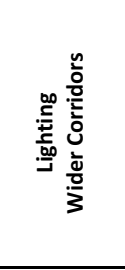 } & C10 & 2 & 15 & 71 & 86 & 17.44 & 82.56 \\
\hline & C11 & 0 & 1 & 87 & 88 & 1.14 & 98.89 \\
\hline & C12 & 0 & 85 & 3 & 88 & 96.59 & 3.41 \\
\hline & C13 & 1 & 4 & 83 & 87 & 4.60 & 95.40 \\
\hline & C14 & 0 & 86 & 2 & 88 & 97.72 & 2.27 \\
\hline & C15 & 1 & 3 & 84 & 87 & 3.45 & 96.55 \\
\hline & C16 & 0 & 86 & 2 & 88 & 97.73 & 2.27 \\
\hline & C17 & 0 & 1 & 87 & 88 & 1.14 & 98.86 \\
\hline & C18 & 0 & 88 & 0 & 88 & 100 & 0 \\
\hline \multirow{9}{*}{ 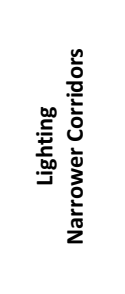 } & C19 & 2 & 71 & 15 & 86 & 82.56 & 17.44 \\
\hline & C2O & 3 & 58 & 27 & 85 & 68.24 & 31.76 \\
\hline & C21 & 0 & 19 & 69 & 88 & 21.60 & 78.41 \\
\hline & C22 & 2 & 62 & 24 & 86 & 72.09 & 27.91 \\
\hline & C23 & 0 & 26 & 62 & 88 & 29.55 & 70.45 \\
\hline & C24 & 1 & 60 & 27 & 87 & 68.97 & 31.03 \\
\hline & C25 & 2 & 25 & 61 & 86 & 29.07 & 70.93 \\
\hline & C26 & 0 & 63 & 25 & 88 & 71.59 & 28.41 \\
\hline & C27 & 1 & 18 & 69 & 87 & 20.69 & 79.31 \\
\hline
\end{tabular}

\subsection{Corridor Width}

To evaluate the first hypothesis (1), data related to the corridors width variable (i.e., C2 to C9) was analyzed by participant. The percentage of choices made by participant favoring the wider corridor considering the width increment (i.e., 50, 100, 150 and $200 \mathrm{~cm}$ ) is presented in Table 2.

The Wilcoxon Signed-Ranks non-parametric test was performed to verify if the percentage of choice for the wider corridors was higher than $50 \%$ (the value $50 \%$ represents the percentage of choice due to chance). Considering the global percentage of choices (regardless of the increment) the Wilcoxon test revealed that the participants made their choices favoring the wider corridors ( $p=.003$, one-tailed). 
Table 2

Results for participants' choices considering the variable width (i.e., corridors C2 - C9) favoring the wider corridors

\begin{tabular}{|c|c|c|c|c|c|}
\hline Participant & $\begin{array}{c}\text { \% } \\
\text { Global }\end{array}$ & \% 50 & \% 100 & \% 150 & \% 200 \\
\hline 1 & 65.63 & 56.25 & 56.25 & 81.25 & 68.75 \\
\hline 2 & 100 & 100 & 100 & 100 & 100 \\
\hline 3 & 96.88 & 93.75 & 100 & 100 & 93.75 \\
\hline 4 & 93.75 & 87.5 & 87.5 & 100 & 100 \\
\hline 5 & 57.81 & 68.75 & 43.75 & 62.5 & 56.25 \\
\hline 6 & 98.48 & 100 & 100 & 100 & 93.75 \\
\hline 7 & 93.75 & 81.25 & 100 & 100 & 93.75 \\
\hline 8 & 17.19 & 12.5 & 12.5 & 37.5 & 6.25 \\
\hline 9 & 100 & 100 & 100 & 100 & 100 \\
\hline 10 & 100 & 100 & 100 & 100 & 100 \\
\hline 11 & 78.13 & 57.14 & 93.75 & 100 & 75 \\
\hline mean & $\mathbf{8 1 . 9 6}$ & $\mathbf{7 7 . 9 2}$ & $\mathbf{8 1 . 2 5}$ & $\mathbf{8 9 . 2 0}$ & $\mathbf{8 0 . 6 8}$ \\
\hline
\end{tabular}

The percentage of choices of the four corridors, width was compared through the Friedman test. This test revealed that the percentage of choices concerning the corridor width were different among the considered corridors $\left(\mathrm{X}^{2}(3)=9.087, p=.021\right)$. Through a non-parametric test for multiple comparisons, it was verified that differences occurred between pairs formed by the corridor with $150 \mathrm{~cm}$ of increment in width and the other increments (i.e., 50, 100 and 200). Table 3 shows the $p$-values for the pairwise comparisons.

Table 3

Multiple comparisons results for the corridor width variable

\begin{tabular}{|c|c|}
\hline Pairwise Comparison & $\boldsymbol{p}$-value \\
\hline 50 vs. 100 & .420 \\
\hline 50 vs. 150 & .047 \\
\hline 50 vs. 200 & 1.000 \\
\hline 100 vs. 150 & .044 \\
\hline 100 vs. 200 & .420 \\
\hline 150 vs. 200 & .007 \\
\hline
\end{tabular}

\subsection{Corridor Lighting}

The percentage of choices favoring the corridors with more lighting (i.e., C10 and C19) by participant, can be seen on Table 4. The data from these corridors were considered to assess the second hypotheses (2). In this way, the Wilcoxon Signed-Ranks test was performed to verify if the percentage of choice made by participants favoring the corridors with more lighting was higher than $50 \%$. For the global percentage of choices (regardless the width increment and light being in the left or in the right corridor), the test revealed that the participants' choice favoring the corridors with more light was statistically higher than $50 \%(p=.004$, one-tailed $)$.
Table 4

Results for participants' choices considering the variable light (i.e., corridors $\mathrm{C} 10$ and $\mathrm{C} 19)$ favoring the corridors with more lighting

\begin{tabular}{|c|c|c|c|}
\hline \multirow{2}{*}{ Participant } & C10 & C19 & \multirow{2}{*}{ \% Global Light } \\
\cline { 2 - 3 } & \% Light right & \% Light left & \\
\hline 1 & 100 & 62.5 & 81.25 \\
\hline 2 & 100 & 87.5 & 93.75 \\
\hline 3 & 100 & 100 & 100 \\
\hline 4 & 100 & 100 & 100 \\
\hline 5 & 87.5 & 50 & 68.75 \\
\hline 6 & 12.5 & 100 & 56.25 \\
\hline 7 & 100 & 100 & 100 \\
\hline 8 & 100 & 100 & 100 \\
\hline 9 & 12.5 & 0 & 6.25 \\
\hline 10 & 100 & 100 & 100 \\
\hline 11 & 100 & 100 & 100 \\
\hline
\end{tabular}

Comparing the percentage of choice for the corridors with more light, independently of the corridor side, the Wilcoxon Signed-Ranks test revealed that the percentage of choice for the lightning corridor was significantly higher than $50 \%$ ( $p=.004$, one-tailed).

The percentage of choices favoring the corridors which were the wider and with more lighting ones (i.e., C11 to $\mathrm{C} 18$ ) were also calculated considering the width increment and the global percentage of choices (Table 5).

The Wilcoxon Signed-Ranks test was performed to evaluate if the global percentage of choices favoring corridors which were wider and with more lighting was higher than $50 \%$. The result allows us to confirm the hypothesis that the participants prefer the corridors which were the wider and with more lighting ( $p<.001$, one-tailed).

The Friedman test was also used to compare the percentage of choices of the four corridors considering the condition wider and with more lighting corridors (i.e., C11 to C18), and there were not verified statistically significant differences among the percentage of choices favoring the different increments of corridor width $\left(\mathrm{X}^{2}(3)=4.714\right.$, $p=.234)$. 
Table 5

Results for participants' choices considering the variables light and width (i.e., corridors $\mathrm{C} 11$ to $\mathrm{C} 18$ ) and favoring the corridors which were the wider and with more lighting

\begin{tabular}{|c|c|c|c|c|c|}
\hline Participant & $\begin{array}{c}\text { \% } \\
\text { Global }\end{array}$ & \% 50 & \% 100 & \% 150 & \% 200 \\
\hline 1 & 100 & 100 & 100 & 100 & 100 \\
\hline 2 & 100 & 100 & 100 & 100 & 100 \\
\hline 3 & 100 & 100 & 100 & 100 & 100 \\
\hline 4 & 100 & 100 & 100 & 100 & 100 \\
\hline 5 & 82.81 & 81.25 & 75 & 81.25 & 93.75 \\
\hline 6 & 98.44 & 100 & 100 & 93.75 & 100 \\
\hline 7 & 100 & 100 & 100 & 100 & 100 \\
\hline 8 & 96.88 & 100 & 93.75 & 93.75 & 100 \\
\hline 9 & 98.44 & 100 & 93.75 & 100 & 100 \\
\hline 10 & 98.44 & 93.75 & 100 & 100 & 100 \\
\hline 11 & 96.88 & 100 & 100 & 100 & 100 \\
\hline mean & $\mathbf{9 7 . 4 4}$ & $\mathbf{9 7 . 7 3}$ & $\mathbf{9 6 . 5 9}$ & $\mathbf{9 7 . 1 6}$ & $\mathbf{9 9 . 4 3}$ \\
\hline
\end{tabular}

\subsection{Corridor width vs. lighting}

Since both hypotheses (i.e., people prefer to follow the wider corridors and the corridors with more lighting) were confirmed, a doubt arouse from this: Which of these two variables is the most influential in the decision-taking about what path to follow?

To answer this question there were also considered a condition where the variables width and lighting were concurrent (i.e., corridors $\mathrm{C} 20$ to $\mathrm{C} 27$ ). For these corridors, the global percentage of choices favoring the wider corridor and the global percentage for the corridor with more lighting were calculated (Table 6). The Wilcoxon Signed-Ranks test results revealed that participants made their choice favoring the corridor with more lighting instead those which were wider ( $p=.043$, one-tailed).

Table 6

Global percentage by participant for choices favoring the wider corridors or the corridors with more lighting when they are concurrent variables (i.e., corridors C20 to C27)

\begin{tabular}{|c|c|c|}
\hline Participant & $\begin{array}{c}\text { \% Global Wider } \\
\text { corridors }\end{array}$ & $\begin{array}{c}\text { \% Global more } \\
\text { light corridors }\end{array}$ \\
\hline 1 & 31.75 & 68.25 \\
\hline 2 & 1.59 & 98.41 \\
\hline 3 & 1.56 & 98.44 \\
\hline 4 & 0 & 100 \\
\hline 5 & 42.19 & 57.81 \\
\hline 6 & 93.75 & 6.25 \\
\hline 7 & 4.69 & 95.31 \\
\hline 8 & 0 & 100 \\
\hline 9 & 100 & 0 \\
\hline 10 & 25.40 & 74.60 \\
\hline 11 & 0 & 100 \\
\hline mean & $\mathbf{2 7 . 3 6}$ & $\mathbf{7 2 . 6 4}$ \\
\hline
\end{tabular}

The hypothesis that people prefer to turn to right in a "T-type" intersection (3) was verified analyzing data related to the corridor $\mathrm{C} 1$. The global percentages of choices favoring the corridor on the right and on the left sides are displayed in Table 7. Table 7

Global percentage by participant for choices favoring the right or left corridors (i.e., corridor $\mathrm{C} 10$ )

\begin{tabular}{|c|c|c|}
\hline Participant & \% Left & \% Right \\
\hline 1 & 6.25 & 93.75 \\
\hline 2 & 0 & 100 \\
\hline 3 & 12.5 & 87.5 \\
\hline 4 & 68.75 & 31.25 \\
\hline 5 & 0 & 100 \\
\hline 6 & 100 & 0 \\
\hline 7 & 50 & 50 \\
\hline 8 & 62.5 & 37.5 \\
\hline 9 & 43.75 & 56.25 \\
\hline 10 & 35.71 & 64.29 \\
\hline 11 & 8.33 & 91.67 \\
\hline mean & $\mathbf{3 5 . 2 5}$ & $\mathbf{6 4 . 7 5}$ \\
\hline
\end{tabular}

The results shown a trend for the participants' choices favoring the right corridor $(64,75 \%)$ which is in line with results found in literature $[5 ; 6]$. However, the results obtained with the Wilcoxon Signed-Ranks test to evaluate if the percentage of choice favoring the right corridors was statistically higher than $50 \%$ did not confirm it ( $p=.115$, one-tailed), pointing that the laterality effect was not verified.

\section{Conclusion}

When people who are unfamiliar with a building are confronted with a situation where they have to choose between two alternative corridors ("T-type" intersection) they could rely in some variables that can be implicit in the overall configuration of the space. In this way, these variables can act as a factor of attractiveness, influencing their displacement within a building. The main objective of this study is to assess the use of two variables as attractiveness factors, the corridor width and the existence of lighting.

For this, an experimental setup was designed using stereoscopic images of virtual environments, where a random sequence of images was presented based on a constant stimulus method. A two-forced choices method was used to collect participants' responses.

Three main hypotheses were formulated: (1) people prefer to follow wider corridors, (2) people 
prefer to follow corridors with more lighting, and (3) people tend to turn right.

Related to the first hypothesis (1), the attained results confirm that people prefer the wider corridors. However, increasing the increment seems do not influence the results. Best results were obtained considering a width increment of $150 \mathrm{~cm}$.

People also prefer to follow by corridors with more lighting, confirming the second hypothesis (2).

Best results were reached when the light and width are summed in the same corridor, resulting in almost $100 \%$ of choices for the corridor with both variables together (i.e., light in a corridor with $4 \mathrm{~m}$ wide $v s$. a corridor with $2 \mathrm{~m}$ wide without lighting).

When the variables light and width are concurrent (i.e., in a "T-type" intersection one alternative corridor has more lighting but is narrower than the other, which is darker and wider), the results shown that people prefer to follow by narrower but with more lighting corridors, allowing to conclude that the variable lighting, for this situation, is a stronger factor of attraction than the variable width.

The third hypothesis was not confirmed. Despite the percentage of people choosing the corridor on the right side being higher than for the left side, there were not verified statistically significant differences. It allows us to conclude that people made their choices about the path to follow in a "T-type" intersection in an emergency situation, randomly.

In this study, participants made their choices based on static images and pressing buttons to express it. Besides, the only force exerted over them that could raise their stress level, typical in an emergency situation, was the reduced time exposition to the stimuli. However, in a real situation, when people move through the hallways within a building they retrieve a large quantity of information of the surround environment. This fact may have influenced the results, since participants were forced to decide based on information acquired from a single point of view. A more ecological approach can be considered in future works, in which participants are able to move along the corridors and make their directional choices based on information gathered from the surround environment that can be useful to grant a better navigational decision.

Acknowledgments. This research was supported by the Portuguese Science and Technology Foundation (FCT) grants (PTDC/PSI-PCO/100148/2008 and SFRH/BD/38927/2007).

\section{References}

[1] Conroy, R., (2001) Spatial navigation in immersive virtual environments, in Faculty of Built Environment, University of London: London, p. 249.

[2] Gibson, J.J., (1986) The Ecological Approach to Visual Perception, Boston: Lawrence Erlbaum Associates, p. 352.

[3] Turvey, M.T., (1992) Affordances and Prospective Control: An Outline of the Ontology. Ecological Psychology, 4(3): p. 173-187.

[4] Stoffregen, T.A., (2003) Affordances as Properties of the Animal-Environment System. Ecological Psychology, 15(2): p. 115-134.

[5] Scharine, A.A. and M.K. McBeath, (2002) Right-Handers and Americans Favor Turning to the Right. Human Factors: The Journal of the Human Factors and Ergonomics Society, 44(2): p. 248-256.

[6] Robinson, E.S., (1933) The Psychology of Public Educations. American Journal of Public Health,. 23(2): p. 123-128.

[7] Teixeira, L., et al. (2010) ErgoVR - An approach for automatic data collection for Ergonomics in Design studies. in International Symposium on Occupational, Safety and Hygene - SHO 2010. Guimarães, Portugal: Portuguese Society of Occupational Safety and Hygiene (SPOSHO). 